\title{
Sharing interim trial results by the Data Safety Monitoring Board with those responsible for the trial's conduct and progress: a narrative review
}

\author{
Victoria Borg Debono, Lawrence Mbuagbaw and Lehana Thabane*
}

\begin{abstract}
Background: Sharing interim data, results or result extrapolations is an important issue that can affect trial integrity. The different ways in which Data Safety Monitoring Boards (DSMBs) share interim results with non-DSMB members and the acceptability of such practices are poorly understood. Our objective was to undertake a narrative review specifically on what kind of interim results, if any, should be shared by the DSMB with non-DSMB members and why.

Methods: We conducted a narrative review using a systematic search strategy of several databases and major health research stakeholders. Literature was included if there was some discussion within the full text about sharing interim trial results with non-DSMB members.

Results: About 79.6\% (129/162) of included citations were based on author's views, 16.7\% (27/162) on research guidelines and 3.7\% (6/162) on surveys. The largest group of citations, 73/162 (45\%), expresses the opinion or argument against sharing interim results with exceptions. Trailing closely, 71/162 (43.8\%) of the included citations support the opinion or argument that interim results should not be shared and should remain confidential with the DSMB. Half of the six surveys support sharing in some capacity, while the other three do not. Eleven circumstances were found that potentially warrant interim result sharing by the DSMB; they relate to (1) usual practices by DSMBs, (2) trial completion threatened, (3) patient safety, (4) regulatory approval and (5) other circumstances. Dominant risks for sharing under these conditions are associated with introducing trial bias.

Discussion/conclusion: There was no majority view in the literature. However, the largest group of citations included express the idea that interim results should remain confidential with the DSMB but also acknowledge circumstances when they could be shared with non-DSMB members. Limitations of this review are that (1) the included literature predominately provides personal perspectives, not evidence, and (2) surveys found globally focus on trial monitoring practices lacking detailed information on what specifically to share, with whom and why. More research is needed with the use of a detailed survey of the clinical trial community focused on DSMB sharing interim results, to better understand and guide DSMB interim result sharing practices.
\end{abstract}

Keywords: Data Safety Monitoring Board, Data Monitoring Committee, Interim data sharing, Narrative review

* Correspondence: thabanl@mcmaster.ca

Department of Health Research Methods, Evidence, and Impact (HEI),

McMaster University, Hamilton, Ontario, Canada 


\section{Background}

The Data Safety Monitoring Board (DSMB) or the Data Monitoring Committee (DMC) is responsible for the stewardship of a trial. This group can help oversee the safety of patients in the trial by looking at unmasked safety or efficacy data to make recommendations to the Steering Committee (SC). They can also oversee, in sequential designs, if the trial results have reached the predefined amount of information needed to finish the trial. Importantly, they also protect the trial from the bias that could be introduced during the trial conduct $[1,2]$.

The case that triggered us to assess further this issue of sharing interim trial information was described by Anand et al. [3] when the funding sponsor asked the SC and DSMB of a cardiovascular trial to provide them with interim adaptive conditional power before approving the request for additional funding requested by the investigators. Adaptive conditional power "is the probability that the trial will reach statistical significance if continued to completion if the difference specified in the trial protocol is true, given the outcome events that have already been observed, and the time remaining to observe additional events among patients who are currently event free" [3, 4]. It is a result extrapolation based on interim relative efficacy results [2]. The DSMB refused to give this information because they considered that sharing adaptive conditional power would unmask the trial's interim results.

When we consider a review done by Grant et al. back in 2005 [5] that globally looks at many issues related to data monitoring and interim analysis, we see that there seems to be an accord that interim results and DSMB deliberations remain confidential. However, the review are also mentions instances where interim results may be shared with the independent unmasked statisticians or other individuals such as the chair of the SC if the DSMB deems it best to do so because of a safety issue. See the Discussion section for a further discussion of this review. Still, the review lacks certain details such as the specifics of what kind of interim results are shared and with whom. Sharing of interim trial data, results or result extrapolations by the DSMB with individuals who are non-DSMB members, who are responsible for the conduct of the trial, can negatively affect trials [6]. One of the major concerns related to sharing interim trial results [1] is the potential for non-DSMB members to consciously or subconsciously introduce bias that will affect the final trial's results [1, 7]. This is an especially important issue for phase III trials, which are usually designed and used to find definitive evidence on efficacy and safety endpoints to inform practice or for regulatory drug approvals [1, 2]. This case [2] and the review [5] brought to mind the following questions: Is it possible that there are other circumstances where the DSMB is justified in sharing interim data, results or extrapolations with non-DSMB members? If so, what information would be shared in such circumstances and with whom?

The overall objective of this review and commentary is to (1) provide a summative narrative review of the views and opinions on the issue of the DSMBs sharing interim results during the conduct of a clinical trial, particularly phase III trials, with the Principal Investigators (PIs), the sponsor, the SC, other parties responsible for the conduct of the trial or any other non-DSMB member(s); and (2) discuss what interim data, results or result extrapolations the DSMB should share, if anything at all, with whom and under what circumstances. The information required to inform this narrative review was gathered from a systematic literature search. For simplicity, the remainder of this review will refer to any assortment of interim data, interim results or interim result extrapolations as interim results. Throughout the rest of the review we will also refer to PIs, the sponsor, the SC, investigators, site managers, independent unmasked statisticians, the funder(s), or patients enrolled in the trial or any other party responsible for the conduct or completion of the trial as non-DSMB members; we will be more specific when needed.

\section{Methods}

A narrative review was considered the most appropriate method to use because it allowed us to explore the literature and inductively evaluate qualitative and quantitative information. We anticipated that most of the literature we would find would be opinions, policies or guidelines, and a narrative review would require an inductive approach for theme analysis and categorisation. To find literature discussing the issue of DSMBs sharing interim results, a broad and comprehensive systematic search of the literature was done in December 2015 within the databases of PubMed (includes all MEDLINE citations), Web of Science, EMBASE and CINAHL from the inception of all four databases using a detailed search strategy for each of them, as outlined in Additional file 1. Key phrases related to 'Data Safety Monitoring Boards' were utilised in each of the four databases as well as a filter for articles in the English language.

The title and abstract for each citation that came up within the search of each of the four databases were reviewed. Citations were eligible and included for full-text review if the title or abstract associated with a particular citation met the following inclusion criteria: [(1) related to DSMB issues OR (2) related to the management, operation, conduct, use, experience, or discussion of DSMBs] AND (3) the article associated with the citation was published in English. Subsequently, citations from the full-text review were eligible and included for full-text information extraction if there was some focused discussion or 
statement, within the full-text, about sharing interim trial results with parties outside of the DSMB. Reference lists from included articles were searched for other unique articles discussing the issue of DSMBs sharing interim trial results using the same inclusion criteria. Additionally, two major textbooks that solely focused on the operation and management of DSMBs were also reviewed and consulted [1,2]. These were found upon discussion with a professor who is a health methodologist with expertise in clinical trials.

We also searched regulatory, governmental and guideline groups from the USA, Canada, UK, European Union and Australia, and two international groups, the International Conference on Harmonisation of Technical Requirements for Registration of Pharmaceuticals for Human Use (ICH) [8] and the World Health Organisation (WHO) [9]. For a list and details of the organisations that were searched directly for relevant literature or information from their respective websites, see Additional file 1. Two strategies were used in combination to find relevant literature on the issue of interim result sharing by the DSMB within major governmental/regulatory/funding bodies and guideline groups (Additional file 1). Documents and webpages on an organisation's website that had information about clinical trial research and DSMBs, as indicated from a webpage's or document's title or within the full text, were eligible and included for full-text information extraction. A full-text review was done immediately because most webpages or documents found on these organisation websites are not structured like most journal articles with an abstract. Documents and pages from the full-text review were eligible and included for full-text information extraction if there was some focused discussion or statement within the full text about sharing interim trial results with parties outside of the DSMB. Please note that within the Results and Discussion sections, not all the citations included from our systematic search of the literature for this review are cited in this paper. A full citation list of articles found to support this review in its entirety is given in Additional file 2. All screening and full-text extraction was done independently by one of the authors (VBD) for this narrative review and then checked by co-reviewers (LM and LT). Information extracted from the included full text pertained to the sharing of interim trial results with parties outside of the DSMB. Any disputes or concerns were resolved by consulting co-reviewers (LM and LT). We worked inductively and thus retrospectively with the included literature to perform a categorisation and thematic literature analysis. Analyst triangulation was used among co-reviewers (LM and LT) with VBD to ensure that categorisation and thematic analysis of literature were sound. No changes were made to the review process that was initially planned. Our review is principled in pragmatism review because we were not initially sure what categories or themes would emerge from the literature. Please see the RAMESES checklist [10] attached as Additional file 3.

\section{Results}

A total of 162 articles, documents, policies, guidelines books or webpages were included for this review. See Fig. 1 for a flow diagram showing the inclusion process and the number of articles included.

There are mixed views and opinions on the issue of the DSMBs sharing interim results during the conduct of a clinical trial with non-DSMB members. Out of the 162 included articles, 129 (79.6\%) were based on author's views, $27(16.7 \%)$ on DSMB or trial research guidelines and $6(3.7 \%)$ on surveys of trialists. The literature falls into three categories of opinions: Category 1 - literature that expresses the opinion or argument against sharing interim results, stating that they should remain confidential with the DSMB (71/162, 43.8\%); Category 2 - literature that expresses the opinion or argument against sharing interim results with exceptions (73/162, 45.0\%); and Category 3 - literature that expresses the opinion or argument in favour of sharing interim results by the DSMB with a non-DSMB group (18/162, 16.8\%). No one group held the outright majority. However, the largest two literature groups that were very close percentage wise were Category 1 (43.8\%) and Category 2 (45.0\%).

Six surveys were also found out of the 162 included articles that looked globally at trial monitoring practice as described in detail in Table 1 . These surveys did not specifically focus on the issue of DSMBs sharing interim results during the conduct of a clinical trial with nonDSMB members. However, all of them asked at least one question that was related to the issue and surveyed those who were somehow involved in trials. Two of the six surveys report results that are quantitatively unclear, as their results are qualitatively described. The remaining four of the six surveys report results quantitatively. The target populations for these surveys varied, ranging from directors of the statistical centres from 12 cancer cooperative groups sponsored by the National Cancer Institute (NCI) in the USA from 1993 [11], trialists of past and ongoing trials [5, 12], PIs and biostatisticians on DSMBs and Institutional Review Board (IRB) community representatives [13], major funders of trials, regulatory agencies and other relevant organisations related to trial research [5]. Methods to obtain the sample also varied. Sampling frames included statistical centres from 12 cancer cooperative groups sponsored by the NCI in the US in 1993 [11], the US National Institutes of Health (NIH) [12], ClinicalTrials.gov, a MEDLINE search of articles pertaining to randomised controlled trials (RCTs) from Biometrics or Statistics in Medicine, the Office of Human Research Protection website [13], a database of 

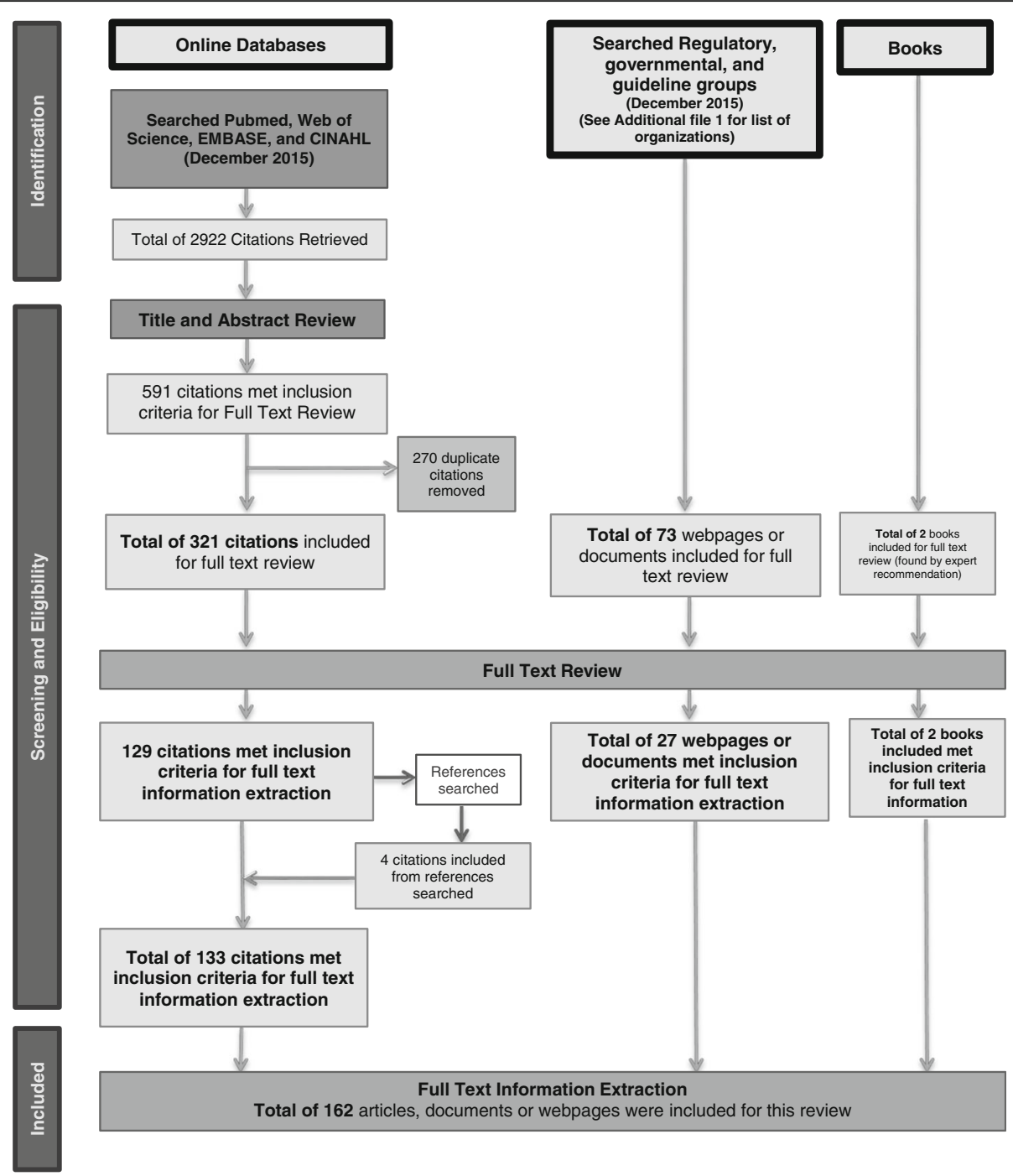

Fig. 1 Flow diagram of the literature inclusion process

Health Technology Assessment (HTA) programme and Medical Research Council (MRC) trials [5] and a list of 25 handpicked organisations that are major funders of trials, regulatory agencies and other relevant organisations related to trial research [5]. Response rates ranged from $40 \%$ to $100 \%[5,11-13]$. The number of responses to these surveys varied from 12 to 309 [5, 11-13]. Respondents were not chosen at random in two cases $[11,12]$. The other four surveys had some method to select respondents at random $[5,13]$. Survey collection methods included telephone interviews [5], email surveys [5] and mail/paper surveys [5, 11-13].

Three of the six surveys $[5,11,13]$ (one qualitatively and two quantitatively reported) support the view against sharing interim results, stating that they should remain confidential with the DSMB (Category 1 view). For one of the two surveys reported quantitatively [11], all respondents $(n=9)$ indicated that NCI groups, at the time the survey was administered, did not provide unmasked outcome reports to the participants. For a second question in the same survey asking about which non-DSMB members had access to interim data reports [11], it was reported that $70 \%$ of the respondents $(n=10)$ indicated that non-DSMB members do not access interim data reports. For the remaining three respondents who answered this question, who alternatively stated that non-DSMB members do have access to interim data reports, there is no mention as to who specifically gets this information and why. For the second survey [13], as can be seen in Table 1, the majority of respondents, all of whom were biostatisticians, PIs or IRB community members, indicate that the sponsor should be masked to interim data or results (percentages can be viewed in Table 1). Masking of other non-DSMB 


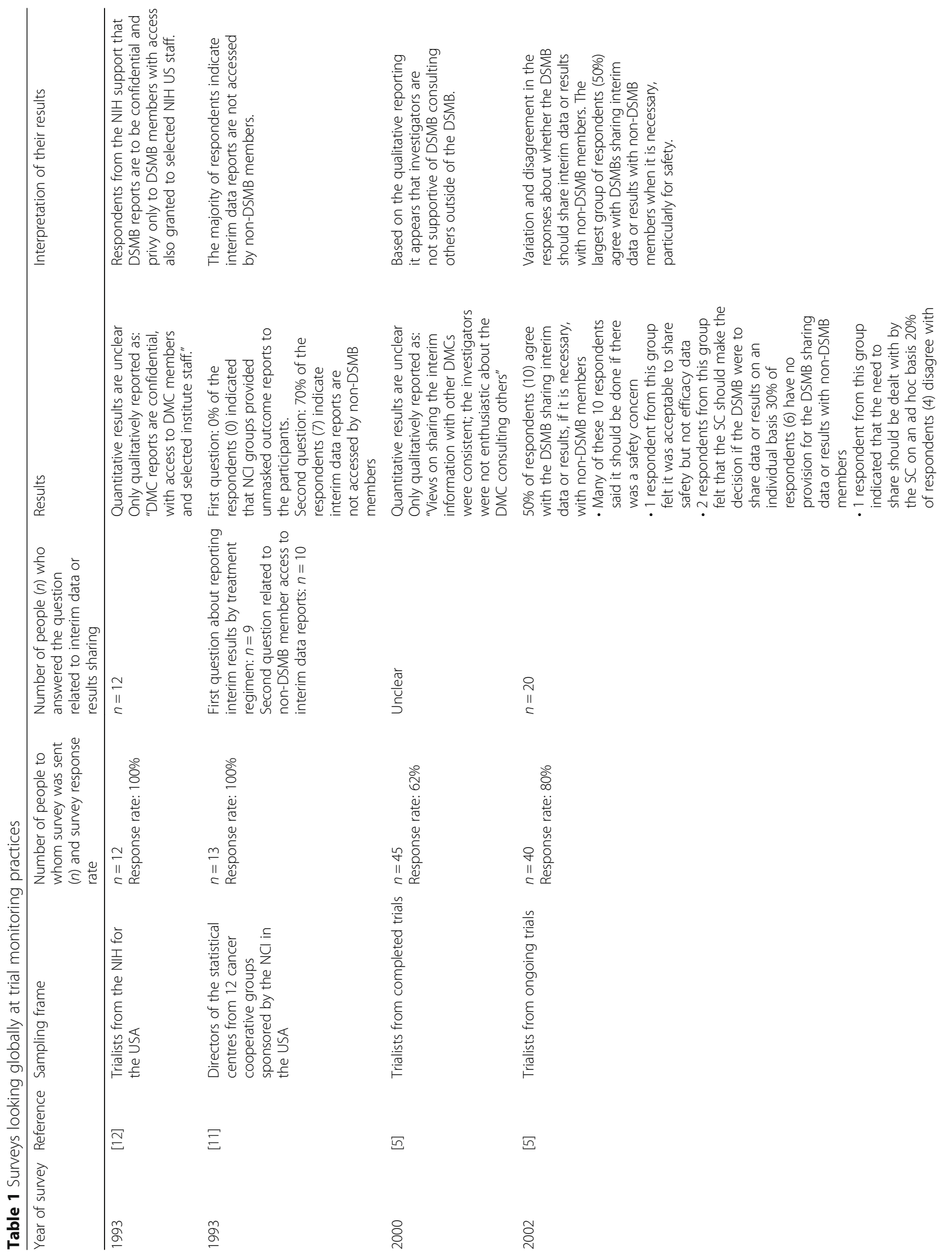



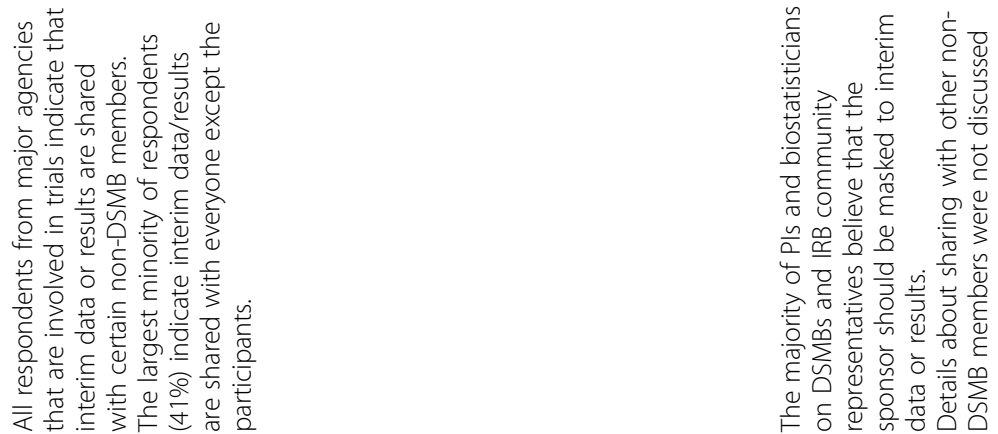

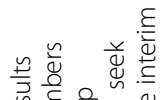

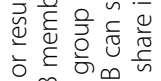

匹 $\sum^{\infty}, \sum^{\infty}$

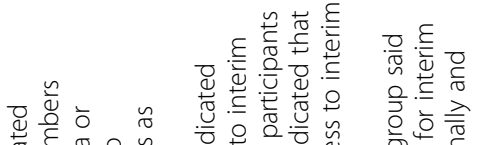

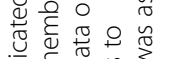

言 $E \frac{\pi}{0} \tilde{u}$

过 0

过

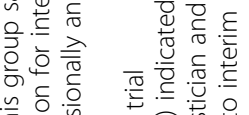

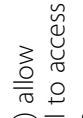

守㝘京

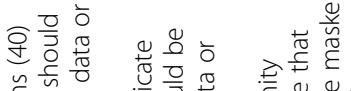

竞

ह

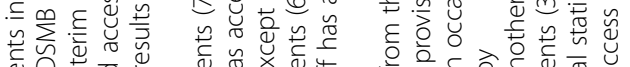

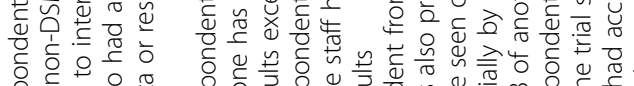

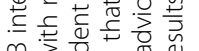

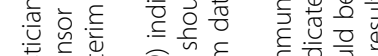

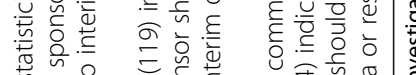

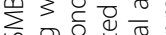

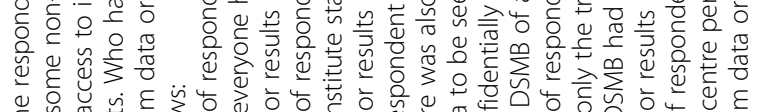

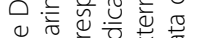

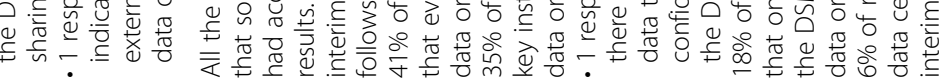

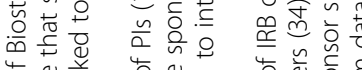

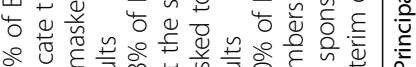

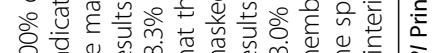

$\underset{\substack{\text { II } \\ \Sigma}}{\stackrel{2}{2}}$

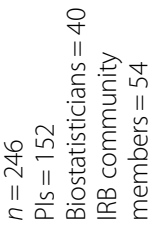

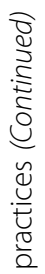

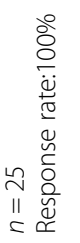

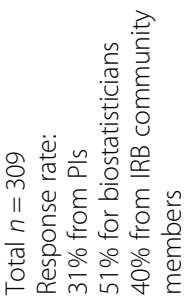

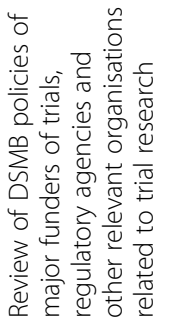

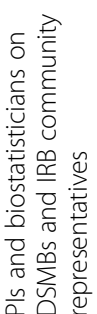

$\sqrt{5}$

$\bar{\Xi}$

文

$\bar{i}$

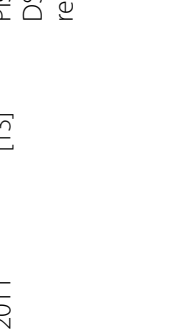


members besides the sponsor was not mentioned. For the minority of respondents who said "No" to this question asking if the sponsor should be masked to interim data or results ( $0 \%$ biostatisticians, $21.7 \%$ PIs and $37.0 \%$ IRB community members), there was no mention as to why this information should be shared with the sponsor. For the one survey that qualitatively reported their results indicating "Views on sharing the interim information with other DMCs were consistent; the investigators were not enthusiastic about the DMC consulting others" [5], there was no mention of whether there were respondents with another view.

One survey [5] (quantitatively reported) supported the Category 2 view: against sharing interim results but with exceptions. The largest group of respondents from this survey, for the question related to interim data or results sharing $(50 \%, 10 / 20)$, agreed with DSMBs sharing interim results with non-DSMB members if it was necessary, particularly for concerns related to participant safety [5]. Specifically with whom this information would be shared was not clear. For the remaining respondents to this question, $30 \%(6 / 20)$ of respondents had no provision for, or idea about, the DSMB sharing interim data or results with non-DSMB members, and 20\% (4/20) of respondents disagreed with the DSMB interim data or results sharing with non-DSMB members.

Two surveys $[5,12]$ (one qualitatively and one quantitatively reported) supported the view in favour of sharing interim results by the DSMB with a non-DSMB group (Category 3 view). For the survey that was reported quantitatively [5], all of the respondents to the question related to interim data or results sharing $(n=17)$ indicated that someone outside of the DSMB had access to interim data or results during their trial. With whom interim results were shared was indicated as follows: $41 \%$ of respondents indicated everyone except the participants, 35\% of respondents indicated key institute staff (one respondent from this group said there was also provision for interim data to be seen occasionally and confidentially by the DSMB of another trial), $18 \%$ of respondents indicated only the trial statistician and the DSMB and 6\% stated that the data centre personnel had access to interim data or results. Why information was shared with these nonDSMB members was not discussed. For the other survey [12] that reported results qualitatively, it was indicated that respondents $(n=12)$ from the US NIH support that DSMB reports are to be confidential and privy only to DSMB members. However, access is also granted to selected US NIH staff, these being non-DSMB members. Why information was shared with selected US NIH staff was not discussed.

For the other 158 documents, which were not describing surveys, we assessed for a time trend to see where the views and policies lie for the last ten years, back to
2006, in regard to the three categories we identified. For the literature in Category 1, dating back to 1981, 54\% $(37 / 68)$ of the literature comes from the past ten years alone. For Category 2, the literature dates back to 1998 . About 24\% (4/17) of the literature comes from the last decade. For the literature in Category 3, dating back to 1991, 52\% (38/73) of the literature comes from the past ten years. The most recent literature, count and percentage wise, has predominately supported the DSMB not sharing or not sharing but with some exceptions. We also found that regulation, policy or guideline documents predominately support Category 3 (55\%, i.e. $15 / 27$ of the 27 regulation, policy or guideline documents included in our review).

In regard to our second objective, there is a subset of the literature within Category 2 or 3 literature that discusses what interim results the DSMB should share, with whom and the circumstance (why). Eleven circumstances that may warrant the DSMB sharing interim results with non-DSMB members are explained in Table 2, generally categorised under four themes: (1) current usual practice by DSMBs, (2) trial completion is threatened, (3) concern about patient safety and (4) regulatory approval). There is also a category for other special circumstances that includes three unique situations for DSMB sharing of interim results that did not fit into a theme. Six of these eleven circumstances are supported in the literature with real-life examples. What is shared by the DSMB with non-DSMB members varies depending on the particular circumstance. For many of the cases where sharing may be warranted, a risk or counter argument to sharing is indicated where applicable. Most of the risks with sharing in these circumstances are predominately associated with introducing bias in the trial that will affect the final trial results. It is indicated in the literature that there is always the potential that sharing interim results with non-DSMB members may do harm to a trial by disturbing equipoise $[14,15]$, as people may make inaccurate impressions about what is happening between treatment groups [3,16-18]. It is explained that when equipoise is disturbed with knowledge of interim results by those operating and managing the trial and those participating in the trial, there may be actions people can take, either consciously or subconsciously [19], that can bias the trial's results $[1,16,19]$. The introduction of bias can reduce the credibility and integrity $[16,20,21]$ of the trial, rendering the results questionable $[16,20,22]$.

\section{Discussion}

\section{What are the findings from the review?}

We found three main views on the DSMB sharing interim results with non-DSMB members. These views were (1) against sharing (Category 1), (2) against sharing interim results with exceptions (Category 2) and (3) in 
Table 2 Circumstances where interim result sharing may be warranted by the DSMB

\begin{tabular}{|c|c|c|c|c|}
\hline Circumstance & $\begin{array}{l}\text { With whom would the } \\
\text { DSMB share? }\end{array}$ & What to share? & Risk or counter argument & Reference \\
\hline
\end{tabular}

Theme 1) Current usual practice by DSMBs

Circumstance 1: When the DSMB recommends early termination and the recommendation needs to be evaluated by the SC and sponsor

Circumstance 2: When the DSMB has concerns about the interim data or results given to them by the unblinded independent statistician or DAC for their interim review

Theme 2) Trial completion is threatened

Circumstance 3: When the trial may have to stop early because of poor accrual due to special circumstances, and it may be possible to improve accrual by sharing interim data or results, when all other efforts to improve accrual are exhausted

Circumstance 4: When there is a need to restore equipoise when one of two related trials finishes first and threatens the completion of the unfinished trial

Theme 3) Concern about patient safety

Circumstance 5: When an uncertain severe safety issue appears at interim in a trial and there is another similar trial still underway

Circumstance 6: When the DSMB assesses the risk of there being a serious adverse event at interim for enrolled patients in a particular treatment group, but
Specified representative(s) of their trial's SC and sponsor

Unmasked interim results

Risk: If the trial were to continue despite the recommendation to terminate, those few individuals privy to the interim data should not be a part of making future trial decisions. This will protect the trial's integrity from potential biasing of results

Trial's independent statistician or DAC

Anything needed

None made

[16, 20, 27-38]

Risk: Risk of biasing trial

The public

Sharing unmasked but limited comparative interim results that will help restore equipose

The DSMB of the similar trial

Safety: Unmasked interim safety result

Trial patients

Safety: Unmasked interim safety results results even when special conditions are met as indicated by Stephens et al. [39]. Sharing interim results should be a judgement call that weights the benefits of sharing against the potential risk of biasing trial results

Risk: Sharing may erode the independence of each trial in regards to the independent confirmation of results

$[16,30,39-43]$

$[39]^{*}$

$[1,16,44]$

$[1]^{*}$

$[1,5,6,17,23,44-52]$

$[1]^{*}$

Risk: Unmasking of interim safety results with the trial patients may risk biasing the trial results, but in some cases it is ethically imperative to let the patients know of the severe
$[1,14,18,53,54]$

$[1]^{*}$ 
Table 2 Circumstances where interim result sharing may be warranted by the DSMB (Continued)

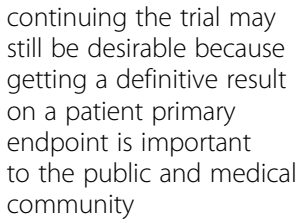

continuing the trial may still be desirable because getting a definitive result on a patient primary endpoint is important to the public and medical community

Theme 4) Regulatory approval

Circumstance 7 : When the The regulators
regulator is currently
assessing licensing
approval for a new drug/
treatment submitted with
results from a completed
trial and there is still a
similar trial underway that
will provide important
new substantial information
regarding results

Circumstance 8: When a The regulators regulatory wants to assess a drug for conditional or accelerated/ expedited approval for a manufacturer to be able to market a drug early

Other special circumstances

Circumstance 9: When adaptive confirmatory trials base interim trial adaptive changes on the trial's interim results

Circumstance 10: When patients outside of the trial are facing important treatment decisions and may benefit from some interim results from non-inferiority or superiority trials with a long follow-up

Circumstance 11: When sponsors, investigators or regulators are planning for future studies, new products or allocating resources for future use
Authorised qualified persons at the sponsor (1 or 2 people) who are not participating in the trial but can assist with trial adaptations

The public and patients and physicians facing important treatment decisions
Relevant unmasked interim results that will help with assessing the status that should be given for a licensing application

Unmasking interim results

Whatever is agreed upon a priori

Relevant unmasked interim results that will help with treatment decision safety risks to allow them to decide whether they want to continue in the trial and before allowing the trial itself to continue
Risk: Interim review of the second ongoing trial could jeopardise its own integrity public could prognosticate the results of that trial based on the regulator's subsequent decision to either approve or delay a manufacturer's licensing application

Risk: Bias could also be introduced to the trial with knowledge of regulatory decisions made based on interim results and known threshold criteria for approval, even if exact interim endpoints are not shared publically

Risk: Unmasking of interim data or results can introduce bias and risk trial integrity

Risk: Knowledge of an interim endpoint result could influence a clinical decision to have a new treatment before safety of that treatment is determined more definitively in ongoing trial and introduce bias, as the

$[1,20]$

$[1]^{*}$

$[1,5,16,28,55-61]$

$[57,60]^{*}$

$[30,62-68]$

$[11,69,70]$

Risk: Bias can be introduced to the unfinished trial if new plans are to be published and can be interpreted by a wider audience. Planning errors could result from using uncertain interim results comparative interim information. This could be:

- Control group event

rates $O R$

- Control group adverse event rates OR

- Pooled event rate

DAC Data Analysis Centre, DSMB Data Safety Monitoring Board, SC Steering Committee

Circumstances 3-8 have a real-life example and an asterisk $\left({ }^{*}\right)$ next to the associated reference(s) with the example

favour of sharing interim results (Category 3). The literature predominately supported Category 1 and Category 2 in similar proportions. We found that the literature in Category 2 and Category 3 presented 11 cogent reasons for sharing interim results by the DSMB with various non-DSMB members in certain circumstances (Table 2). The three surveys $[5,12]$ that support the Category 2 and Category 3 views do not specifically indicate what should or should not be shared in circumstances that may warrant sharing interim results. However, one of the surveys [5] that support Category 2 indicates that the DSMB having a safety concern is a circumstance that justifies the DSMB sharing interim results with nonDSMB members. In these 11 circumstances, what is shared and with whom depends on the circumstance. Six of the 11 circumstances (see Table 2) have real-life 
examples (anecdotal evidence) where interim result sharing by the DSMB with a non-DSMB member helped the trial and the patients who were enrolled. However, for most of these 11 circumstances, there are risks acknowledged-mainly in regard to introducing bias that may affect the trial's final results. Based on these 11 circumstances that may occur, there is possible legitimacy in the notion that what may or may not be shared and with whom at interim should be a judgement call made by a trial's DSMB, where the DSMB as a group balances the apparent risks and benefits in those circumstances that arise regarding the need to protect both the safety of the patients enrolled and the trial's integrity. Sometimes the DSMB may find that the benefits of sharing seem to outweigh the risks, as was illustrated by some real-life examples that supported 6 of the 11 circumstances. The DSMB may also find that the risks of sharing are not worth the benefits that could result. The opinion of the DSMB sharing interim results is also supported in part by both Chalmers et al. [23] and Shah et al. [24]. Chalmers et al. [23] comment on the need to share interim results when the occasion is appropriate and to identify and plan for such situations a priori when possible.

How do these findings compare with those of similar works? While our review is unique in that it solely focuses on the issues of interim result sharing by the DSMB with non-DSMB members, another review, done earlier in 2005 by Grant et al. [5] under the auspices of the National Health Service (NHS) in the UK, looks globally at many issues related to data monitoring and interim analysis. One of 23 questions they ask addresses in part the issue with DSMB confidentiality of interim data. They found within their literature review under Question 8: Should the DMC deliberations be open or closed (confidential or secret as opposed to publicly available)? that "There is near unanimity that the interim data and the deliberations of the DMC should be absolutely confidential" [5], which supports what we found in our review with Category 1 literature which held a large percentage of the literature reviewed (43.8\%). However, a subquestion to their Question 8 asked: Who outside the committee should see the interim analysis and how is this changed by whether the analyses were blinded or unblinded? They indicate that some have suggested that an independent unmasked statistician should see the interim analysis [5], which is what we found for Circumstance 2 described in our Table 2. They also indicate that the DSMBs may allow certain individuals such as the chair of the SC to become unmasked to certain interim results, especially if this is deemed by the DSMB of the trial to serve patient/participant safety best [5]. For Circumstances 5 and 6 in Table 2, we also find that a severe safety issue in the trial is a potential driving factor to share unmasked interim safety results with nonDSMB members. Thus, their review [5] also supports what we found in Category 2 literature: even though interim results should remain confidential with the DSMB, there are circumstances the DSMB must consider that could warrant interim sharing outside of the DSMB. Our review goes into detail about the circumstances that may warrant sharing (see Table 2), and we do our best to summarise all the views on this issue. Our intent was also to discuss what to share and with whom in those circumstances and, as described above, we found that it greatly depends on the circumstance in which the DSMB finds itself, in regard to the trial.

\section{What are the key limitations?}

One limiting factor of this review is that the majority of the included literature was based on personal perspectives (79.6\%). Though these perspectives brought up important points, these views represent a small fraction of all the professionals who are involved in trials and are not based on evidence. Many of these views contributed to describing the 11 circumstances found in Table 2, where only 6 out of the 11 circumstances were supported by anecdotal evidence. The other 7 circumstances were based on the author's experience or view that would warrant sharing by the DSMB if the DSMB deemed it necessary and safe to do so.

Another limitation is that the six surveys do not fully help us with our objective to understand, with empirical evidence, what interim results, if any, the DSMB should share, with whom and under what circumstances. They globally focus on trial monitoring practices and not in depth or specifically on the issue of DSMB sharing interim results. At most, one or two questions within each survey ask a question related to the DSMB sharing interim results with other non-DSMB groups, but the questions are not asked in a consistent way for all the surveys. For instance, for three of these surveys, the questions related to interim result sharing asks respondents about their current practices regarding who has access to unmasked outcome reports $[5,11,12]$ at their research institutions. For another survey, the question very specifically asks respondents if the sponsor should be masked to interim results [13]. For two of the surveys [5], the question related to interim result sharing asks respondents if they think unmasked interim results should be shared with non-DSMB members. So, although we can understand overall where support lies from each survey in regard to sharing interim results, and in some surveys we have a bit of information about with whom interim results may be shared, it is still extremely unclear what specific kind of interim result should be shared, with whom that result should be 
shared and for what reason or circumstance. There is not one survey that consistently asks the same group of respondents who are trialists, what interim results specifically should be shared, with a direct follow-up question about who outside of the DSMB should share that specific interim result and for what reason (why). This is a complex topic. It is challenging for any survey soliciting responses about global trial monitoring practices to use one or two questions to sufficiently address and provide enough information clarifying what interim results the DSMB should share, if anything at all, with whom, and why. Multiple questions or an entire survey dedicated to the topic of DSMB interim result sharing is needed.

\section{Implications of the findings}

Our findings inform trialists and those who enact trial policies and guidelines that there are mixed views on the DSMB sharing interim results with non-DSMB members. We could argue that out of the three categories, the literature in Categories 1 and 2 dominates in that the DSMB should not share interim results with nonDSMB members, but the literature in Category 2 suggests that there may exceptions. The exceptions include 11 possible circumstances as described in Table 2. However, the findings from this review need to be substantiated with more research. The empirical evidence found within three of the six surveys $[5,13]$ suggests there is support for sharing interim results with certain nonDSMB members, but the details on what specifically should be shared and for what reason are unclear. Based on the limitations described in the previous section, more empirical evidence is needed to clarify specifically what interim results should or should not be shared by the DSMB with non-DSMB members, with whom and for what reason or circumstance, to better inform monitoring practices, policies and guidelines that protect the safety of the participants enrolled and trial validity.

For the situation described earlier by Anand et al. [3], we also question: How useful is it to share unmasking yet non-comparative interim results (e.g. control group event rates without knowledge of the pooled events rates)? Also, how useful is it to share results that appear masking of comparative results (e.g. adaptive conditional power or aggregate/pooled results by treatment group)? It is thought that knowledge of aggregate/pooled results can lead to concerns about making assumptions about interim results $[25,26]$ that are not necessarily true, which could lead to introducing bias in the trial. Knowledge of such potentially unmasking information, such as the adaptive conditional power, could jeopardise the integrity of the trial, as it does indicate the probability of the trial showing a favourable significant result [2]. In the case described by Anand et al. [3], the request for the adaptive conditional power by the trial sponsor was denied by the trial's DSMB, and the decision to not share was additionally supported by the trial's SC, PI and others outside of the trial who were consulted. There was also mention of the DSMB sharing an 'unconditional' conditional power with the sponsor. This 'unconditional' conditional power calculation [3] was shared with the sponsor because it is thought to mask the efficacy results if given out at interim, but also provide reassurance to the sponsor that the trial will have the power to answer the primary hypothesis initially set out at the design stage of the trial, when the trial is completed [3]. Is providing the 'unconditional' conditional power a helpful alternative to sharing aggregate/pooled results? Should a result extrapolation such as aggregate/pooled results or adaptive conditional power be shared? How is such information interpreted? The issue of sharing aggregate/pooled interim results needs further investigation. More clarity is also required on the specifics of sharing aggregate/ pooled interim results, particularly interim results that are thought to be masking, such as the combined event rate, and result extrapolations, such as adaptive conditional power, that have been requested in the past [3].

\section{Conclusions}

Interim result sharing is an important issue because it affects the validity of the results from confirmatory trials on which we base regulatory and practise decisions, impacting the health and lives of many. From this review, two categories of the literature dominate (Category 1 and Category 2), but not in majority as distinct groups. Category 1 is against sharing interim results, stating that they should remain confidential with the DSMB, and Category 2 shares the same sentiment as Category 1 but additionally acknowledges exceptions, that there are circumstances that may warrant the DSMB to share interim results with certain non-DSMB groups/members. What is shared with these non-DSMB members depends on what the situation calls for and should be assessed by the DSMB using their expertise to balance risk(s) with the potential benefit(s) regarding participant safety and trial validity and integrity. Because of the limitations of the evidence found, collecting more empirical evidence through a survey of the general clinical trials community focused on the issue of DSMB sharing interim results (what, if any, interim result(s) to share, with whom and under what circumstance) is needed to better understand and guide DSMB interim information sharing practices.

\section{Additional files}

Additional file 1: Search strategies for literature. (DOCX 33 kb)

Additional file 2: Citations of included articles for review. (DOCX $33 \mathrm{~kb}$ )

Additional file 3: RAMESES checklist. (DOCX $18 \mathrm{~kb}$ ) 


\section{Abbreviations}

CRO: Contract Research Organisation; DAC: Data Analysis Centre; DMC: Data Monitoring Committee; DSMB: Data Safety Monitoring Board; FDA: Food and Drug Administration; IRB: Institutional Review Board; ISC: Independent Statistical Centre; NCl: National Cancer Institute; NHS: National Health Service; $\mathrm{NIH}$ : National Institutes of Health; PI: Principal Investigator; RCT: Randomised controlled trial; SC: Steering Committee; SDAC: Statistical Data Analysis Centre; SOP: Standard Operating Procedure; UK: United Kingdom

\section{Acknowledgements}

Not applicable.

\section{Funding}

The project received full support from the Canadian Institutes for Health Research (CIHR).

\section{Availability of data and materials}

Not applicable.

\section{Authors' contributions}

VBD was involved in all aspects of this study from study design and conception, acquisition of information, information synthesis and writing of the manuscript. LM was substantially involved in supervision of study design and critical revisions to this paper. LT was substantially involved in primary supervision, study design and conception and critical revisions to this paper. All authors read and approved the final manuscript.

\section{Authors' information}

Not applicable.

\section{Competing interests}

The authors declare that they have no competing interests.

\section{Consent for publication}

Not applicable.

\section{Ethics approval and consent to participate} Not applicable.

\section{Publisher's Note}

Springer Nature remains neutral with regard to jurisdictional claims in published maps and institutional affiliations.

Received: 19 October 2016 Accepted: 21 February 2017

Published online: 09 March 2017

\section{References}

1. Ellenberg SS, Fleming TR, DeMets DL. Data monitoring committees in clinical trials: a practical perspective. Hoboken: John Wiley \& Sons; 2003.

2. Herson J. Data and safety monitoring committees in clinical trials. Boca Raton: Chapman \& Hall/CRC/Taylor \& Francis; 2009

3. Anand SS, Wittes J, Yusuf S. What information should a sponsor of a randomized trial receive during its conduct? Clin Trials. 2011;8:716-9.

4. Friedman LM, Furberg C, DeMets DL. Fundamentals of clinical trials. 3rd ed. New York: Springer; 1998.

5. Grant AM, Altman DG, Babiker AB, Campbell MK, Clemens FJ, Darbyshire JH, Elbourne DR, McLeer SK, Parmar MK, Pocock SJ, et al. Issues in data monitoring and interim analysis of trials. Health Technol Assess. 2005;9:1-238. iii-iv.

6. Borer JS, Gordon DJ, Geller NL. When should data and safety monitoring committees share interim results in cardiovascular trials? JAMA. 2008;299:1710-2.

7. Bloudoff-Indelicato M. Threat of interim data leaks prompts call for international rules. Nat Med. 2015;21:200.

8. International Conference on Harmonisation of Technical Requirements for Registration of Pharmaceuticals for Human Use. http://www.ich.org/home. html. Accessed Dec 2015.

9. Who we are. 2017. http://www.who.int/about/who-we-are/en/. Accessed Dec 2015.

10. Wong G, Greenhalgh T, Westhorp G, Buckingham J, Pawson R. RAMESES publication standards: meta-narrative reviews. BMC Med. 2013;11(1):20.
11. George SL. A survey of monitoring practices in cancer clinical trials. Stat Med. 1993;12(5-6):435-50.

12. Geller NL, Stylianou M. Practical issues in data monitoring of clinical trials: summary of responses to a questionnaire at NIH. Stat Med. 1993;12(5-6): 543-51. discussion 553.

13. Tereskerz PM, Guterbock TM, Kermer DA, Moreno JD. An opinion and practice survey on the structure and management of data and safety monitoring boards. Account Res. 2011;18:1-30.

14. Silverman $\mathrm{H}$. Ethical issues during the conduct of clinical trials. Proc Am Thorac Soc. 2007:4:180-4. discussion 184.

15. Slutsky AS, Lavery JV. Data safety and monitoring boards. N Engl J Med. 2004:350(11):1143-7.

16. Fleming TR, Sharples K, McCall J, Moore A, Rodgers A, Stewart R. Maintaining confidentiality of interim data to enhance trial integrity and credibility. Clin Trials. 2008:5:157-67.

17. Rouse DJ. Data monitoring and safety committees and their operations. Obstet Gynecol Surv. 2003:58(5):329-36.

18. Migrino RQ, Topol EJ. A matter of life and death? The Heart Protection Study and protection of clinical trial participants. Control Clin Trials. 2003; 24(5):501-5

19. Hemmings $R$, Day $S$. Regulatory perspectives on data safety monitoring boards: protecting the integrity of data. Drug Saf. 2004;27:1-6.

20. US Food and Drug Administration. The establishment and operation of clinical trial data monitoring committees for clinical trial sponsors. Rockville: US Food and Drug Administration; 2006. p. 38.

21. Eckstein L. Building a more connected DSMB: better integrating ethics review and safety monitoring. Account Res Policies Qual Assur. 2015;22(2):81-105.

22. International Council for Harmonisation of Technical Requirements for Pharmaceuticals for Human Use (ICH). ICH Harmonised tripartite guideline; statistical principles for clinical trials E9. Geneva: ICH; 1998. p. 39.

23. Chalmers I, Altman DG, McHaffie H, Owens N, Cooke RW. Data sharing among data monitoring committees and responsibilities to patients and science. Trials. 2013;14:102

24. Shah SK, Dawson L, Dixon DO, Lie RK. Should sponsors and DSMBs share interim results across trials? J Acquir Immune Defic Syndr. 2011;58(5):433-5.

25. US Food and Drug Administration. FDA guidance on clinical trial data monitoring committees (DMCs). Rockville: US Food and Drug Administration; 2001. p. 237.

26. Pocock SJ. Statistical and ethical issues in monitoring clinical trials. Stat Med. 1993;12(15-16):1459-69. discussion 1471-5.

27. Chow SC, Corey R, Lin M. On the independence of data monitoring committee in adaptive design clinical trials. J Biopharm Stat. 2012;22(4):853-67.

28. Fleming TR, Hennekens CH, Pfeffer MA, DeMets DL. Enhancing trial integrity by protecting the independence of data monitoring committees in clinical trials. J Biopharm Stat. 2014:24(5):968-75.

29. D'Agostino Sr RB. The statistician and the data monitoring committee. Stat Med. 2004:23(10):1501-2.

30. Fleming TR. Standard versus adaptive monitoring procedures: a commentary. Stat Med. 2006:25(19):3305-12. discussion 3313-4, 3326-47.

31. Siegel JP, O'Neill RT, Temple R, Campbell G, Foulkes MA. Independence of the statistician who analyses unblinded data. Stat Med. 2004;23(10):1527-9.

32. NHLBI policy for data and safety monitoring of extramural clinical studies. http://www.nhlbi.nih.gov/research/funding/human-subjects/data-safetymonitoring-policy. Accessed Dec 2015.

33. Guidelines for $\mathrm{NCClH}$-appointed data and safety monitoring boards. https:// nccih.nih.gov/research/policies/datasafety. Accessed Dec 2015.

34. Sartor O, Halabi S. Independent data monitoring committees: an update and overview. Urol Oncol. 2015;33(3):143-8.

35. Williams GW, Davis RL, Getson AJ, Gould AL, Hwang IK, Matthews H, Shih WCJ, Snapinn SM, Waltonbowen KL. Monitoring of clinical trials and interim analyses from a drug sponsors point of view. Stat Med. 1993;12(5-6):481-92.

36. DeMets D, Califf R, Dixon D, Ellenberg S, Fleming T, Held P, Julian D, Kaplan $R$, Levine $R$, Neaton J, et al. Issues in regulatory guidelines for data monitoring committees. Clin Trials. 2004;1(2):162-9.

37. Delgado-Herrera L, Anbar D. A model for the interim analysis process: a case study. Control Clin Trials. 2003:24:51-65.

38. DeMets DL, Fleming TR. The independent statistician for data monitoring committees. Stat Med. 2004:23(10):1513-7.

39. Stephens RJ, Langley RE, Mulvenna P, Nankivell M, Vail A, Parmar MK. Interim results in clinical trials: do we need to keep all interim randomised clinical trial results confidential? Lung Cancer. 2014;85(2):116-8. 
40. Green S, Crowley J. Data monitoring committees for Southwest Oncology Group clinical trials. Stat Med. 1993;12(5-6):451-5.

41. Lilford RJ, Braunholtz D, Edwards S, Stevens A. Monitoring clinical trials interim data should be publicly available. Br Med J. 2001;323(7310):441-2.

42. Miller FG, Wendler D. Is it ethical to keep interim findings of randomised controlled trials confidential? J Med Ethics. 2008;34:198-201.

43. Vail A, Hornbuckle J, Spiegelhalter DJ, Thornton JG. Prospective application of Bayesian monitoring and analysis in an 'open' randomized clinical trial. Stat Med. 2001;20(24):3777-87.

44. Fleming TR, Ellenberg S, DeMets DL. Monitoring clinical trials: issues and controversies regarding confidentiality. Stat Med. 2002;21(19):2843-51.

45. Glover JM, Kay R. Who advises the Data Monitoring Committee (DMC)? A review of regulatory guidance for sponsors on DMCs after 5 years and advice for DMC members. Drug Inf J. 2012;46(5):525-31.

46. Califf RM, Ellenberg SS. Statistical approaches and policies for the operations of Data and Safety Monitoring Committees. Am Heart J. 2001;141(2):301-5.

47. Hicks LK, Laupacis A, Slutsky AS. A primer on data safety monitoring boards: mission, methods, and controversies. Intensive Care Med. 2007;33(10):1815-8.

48. DeMets DL. Relationships between Data Monitoring Committees. Control Clin Trials. 2000;21(1):54-5.

49. Dixon DO, Lagakos SW. Should data and safety monitoring boards share confidential interim data? Control Clin Trials. 2000;21:1-6. discussion 54-55.

50. Dixon DO, Weiss S, Cahill K, Fox L, Love J, McNamara J, Soto-Torres LE. Data and safety monitoring policy for National Institute of Allergy and Infectious Diseases clinical trials. Clin Trials. 2011;8:727-35.

51. Yusuf, Whitley R, Assenzo R, Fleming T, Deykin, Hawkins B, Meier, Gent, Tognoni, Ellenberg J, et al. The operation of data monitoring committees discussion. Stat Med. 1993;12(5-6):527-42.

52. Ellenberg SS. Independent data monitoring committees: rationale, operations and controversies. Stat Med. 2001;20:2573-83.

53. Wittes J, Barrett-Connor E, Braunwald E, Chesney M, Cohen HJ, Demets D, Dunn L, Dwyer J, Heaney RP, Vogel V, et al. Monitoring the randomized trials of the Women's Health Initiative: the experience of the Data and Safety Monitoring Board. Clin Trials. 2007:4:218-34

54. Peppercorn J, Buss WG, Fost N, Godley PA. The dilemma of data-safety monitoring: provision of significant new data to research participants. Lancet. 2008;371(9611):527-9.

55. Fleming TR. Protecting the confidentiality of interim data: addressing current challenges. Clin Trials. 2015;12(1):5-11.

56. Nissen SE. Commentary: confidentiality of interim trial data-the emerging crisis. Clin Trials. 2015;12(1):15-7.

57. US Food and Drug Administration, Department of Health and Human Services, Parks M, Rosebraugh C, Jenkins J, Woodcock J. Memorandum; disclosure of interim cardiovascular risk study data NDA 22271 Nesina (alogliptin) tablets and its fixed dose combination product NDAs 22426 and 203414. Rockville: US Food and Drug Administration; 2013.

58. US Food and Drug Administration. Presentations for: Part 15 public hearing; confidentiality of interim results in cardiovascular (CV) outcomes safety trials. Rockville: US Food and Drug Administration; 2014.

59. London AJ. Commentary: data monitoring confidentiality and FDA transparency. Clin Trials. 2015;12(1):12-4

60. US Food and Drug Administration. Transcript for hearing: Part 15 public hearing; confidentiality of interim results in cardiovascular (CV) outcomes safety trials. Rockville: US Food and Drug Administration; 2014.

61. Confidentiality of Interim Results in Cardiovascular (CV) Outcomes Safety Trials; Part 15 - PUBLIC HEARING BEFORE THE COMMISSIONER; Request for Comments. http://www.fda.gov/Drugs/NewsEvents/ucm405023.htm. Accessed Dec 2015.

62. Gallo P. Operational challenges in adaptive design implementation. Pharm Stat. 2006:5(2):119-24.

63. Gallo P, Chuang-Stein C, Dragalin V, Gaydos B, Krams M, Pinheiro J. Adaptive designs in clinical drug development-an Executive Summary of the PhRMA Working Group. J Biopharm Stat. 2006;16(3):275-83. discussion 28591, 293-8, 311-2.

64. Gallo P. Confidentiality and trial integrity issues for adaptive designs. Drug Inf J. 2006;40(4):445-50.

65. Herson J. Coordinating data monitoring committees and adaptive clinical trial designs. Drug Inf J. 2008;42(4):297-301.

66. Antonijevic Z, Gallo P, Chuang-Stein C, Dragalin V, Loewy J, Menon S, Miller ER, Morgan CC, Sanchez M. Views on emerging issues pertaining to data monitoring committees for adaptive trials. Ther Innov Regul Sci. 2013;47(4):495-502.
67. Day S. Data monitoring committees in clinical trials: best practice, complexities and considerations. Clin Invest. 2015;5(7):615-7.

68. Gallo P, Fardipour P, Dragalin V, Krams M, Littman GS, Bretz F. Data monitoring in adaptive dose-ranging trials. Stat Biopharm Res. 2010;2(4):513-21.

69. Korn EL, Hunsberger S, Freidlin B, Smith MA, Abrams JS. Preliminary data release for randomized clinical trials of noninferiority: a new proposal. J Clin Oncol. 2005;23:5831-6.

70. Allardyce RA, Bagshaw PF, Frampton CM, Frizelle FA, Hewett PJ, Rieger NA, Smith JS, Solomon MJ, Stevenson AR. Ethical issues with the disclosure of surgical trial short-term data. ANZ J Surg. 2011;81(3):125-31.

71. DeMets DL. Commentary on Anand et al. Clin Trials. 2011:8:722-3. discussion 726

72. Rockhold FW, Enas GG. Data monitoring and interim analyses in the pharmaceutical industry: ethical and logistical considerations. Stat Med. 1993;12(5-6):471-9.

73. DeMets DL, Fleming TR, Whitley RJ, Childress JF, Ellenberg SS, Foulkes M, Mayer KH, O'Fallon J, Pollard RB, Rahal J, et al. The data and safety monitoring board and acquired immune deficiency syndrome (AIDS) clinical trials. Control Clin Trials. 1995;16:408-21.

74. Smith MA, Ungerleider RS, Korn EL, Rubinstein L, Simon R. Role of independent data-monitoring committees in randomized clinical trials sponsored by the National Cancer Institute. J Clin Oncol. 1997;15(7):2736-43.

\section{Submit your next manuscript to BioMed Central and we will help you at every step:}

- We accept pre-submission inquiries

- Our selector tool helps you to find the most relevant journal

- We provide round the clock customer support

- Convenient online submission

- Thorough peer review

- Inclusion in PubMed and all major indexing services

- Maximum visibility for your research

Submit your manuscript at www.biomedcentral.com/submit
C Biomed Central 